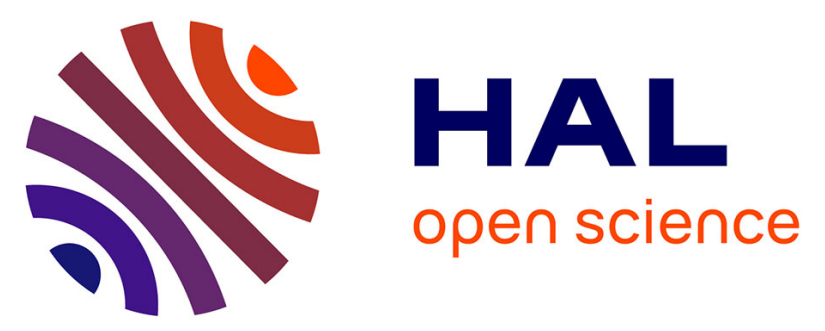

\title{
Sparse SVD Method for High-Resolution Extraction of the Dispersion Curves of Ultrasonic Guided Waves
}

Kailiang Xu, Jean-Gabriel Minonzio, Dean Ta, Bo Hu, Weiqi Wang, Pascal Laugier

\section{- To cite this version:}

Kailiang Xu, Jean-Gabriel Minonzio, Dean Ta, Bo Hu, Weiqi Wang, et al.. Sparse SVD Method for High-Resolution Extraction of the Dispersion Curves of Ultrasonic Guided Waves. IEEE Transactions on Ultrasonics, Ferroelectrics and Frequency Control, 2016, 63 (10), pp.1514 - 1524. 10.1109/TUFFC.2016.2592688 . hal-01386617

\section{HAL Id: hal-01386617 https: / hal.sorbonne-universite.fr/hal-01386617}

Submitted on 24 Oct 2016

HAL is a multi-disciplinary open access archive for the deposit and dissemination of scientific research documents, whether they are published or not. The documents may come from teaching and research institutions in France or abroad, or from public or private research centers.
L'archive ouverte pluridisciplinaire HAL, est destinée au dépôt et à la diffusion de documents scientifiques de niveau recherche, publiés ou non, émanant des établissements d'enseignement et de recherche français ou étrangers, des laboratoires publics ou privés. 
2 Sparse SVD Method for High Resolution Extraction of the Dispersion Curves of Ultrasonic Guided 3 Waves

$5 \quad$ Kailiang $\mathrm{Xu}^{1,2}$, Jean-Gabriel Minonzio ${ }^{2}$, Dean $\mathrm{Ta}^{1,3,4}$, Bo Hu${ }^{1}$, Weiqi Wang ${ }^{1}$, Pascal Laugier ${ }^{2}$

71 Department of Electronic Engineering Fudan University, Shanghai, China.

82 Sorbonne Universités, UPMC Univ Paris 06, CNRS, INSERM, Laboratoire d'Imagerie Biomédicale

9 (LIB), 75006, Paris, France.

103 State Key Laboratory of ASIC and System, Fudan University, Shanghai, China

$4313 *$ *Corresponding authors: E-mails: Kailiang Xu, xukl.fdu@gmail.com 
1 Abstract - The two-dimensional Fourier transform (2D-FT) analysis of multichannel signals is a

2 straightforward method to extract the dispersion curves of guided modes. Basically, the time signals

3 recorded at several positions along the waveguide are converted to the wavenumber-frequency space, so

4 that the dispersion curves (i.e., the frequency-dependent wavenumbers) of the guided modes can be

5 extracted by detecting peaks of energy trajectories. In order to improve the dispersion curves extraction of

6 low amplitude modes propagating in cortical bone, a multi-emitter and multi-receiver transducer array has

7 been developed together with an effective singular vector decomposition (SVD) based signal processing

8 method. However, in practice, the limited number of positions where these signals are recorded results in

9 a much lower resolution on the wavenumber axis than on the frequency axis. This prevents a clear identification of overlapping dispersion curves. In this study, a sparse SVD (S-SVD) method, which combines the SNR improvement of the SVD-based approach with the high wavenumber resolution advantage of the sparse optimization, is presented to overcome the above mentioned limitation. Different penalty constraints, i.e., $l_{1}$-norm, Frobenius norm and revised Cauchy norm, are compared with the sparse characteristics. The regularization parameters are investigated with respect to the convergence property and wavenumber resolution. The proposed S-SVD method is investigated using synthetic wideband signals, experimental data obtained from a bone-mimicking phantom and from an ex-vivo human radius. The analysis of the results suggests that the S-SVD method has the potential to significantly enhance the wavenumber resolution and to improve the extraction of the dispersion curves. 


\section{INTRODUCTION}

2 The dispersion characteristics of elastic guided waves have attracted considerable attention and brought out 3 many useful applications, such as seismic waves analysis [1-6], underwater acoustics [7-9], non-destructive 4 evaluation [10-13] and biomedical applications [14-17]. Following the different implementations of signal 5 recording, the dispersion characteristics extraction methods can mainly be classified into two categories, 6 i.e., single-channel processing [7-9, 11, 14] and multichannel processing [4, 10, 15, 18-20].

7 Regarding the single-channel processing, the time-frequency representation (TFR) method enables the 8 computation of the dispersive energy simultaneously in time and frequency [21]. In 1999, Prosser et al. [11] 9 applied the TFR method to characterize Lamb modes dispersion. Several TFR-based dispersion curves extraction strategies have been proposed. For example, aiming to overcome the TFR uncertainty principle (which actually determines that there is an inherent trade-off between the time and frequency resolution in the spectrogram) and to enhance the mode extraction capabilities, some improved TFR methods have been proposed in which the signals are decomposed into TFR atoms whose group delays are nonlinearly modulated in frequency and determined with respect to the local wave dispersion, such as the group delay shift covariant quadratic TFR [7], warped TFR method [22], Chirplet transform [23], generalized warblet transform based TFR method [24] and dispersion-based TFR method [25]. Recently, Xu et al. [26] have employed the dispersion compensation technique $[27,28]$ for multimode separation. In underwater acoustics field, Bonnel et al. [9] successfully utilized the robust physical a priori information of the oceanic waveguide to separate some overlapped modes, which was difficult with the classical TFR methods. Those refined dispersion mode extraction methods were basically implemented based on artificially 
1 shifting/compensating the mode energy distribution by considering that the dispersion characteristics can

2 be well determined by the modal theory. Since their performances rely on the a priori knowledge of the

3 waveguide characteristics, further improvements are still required. An iterative estimation method has been

4 designed with the dispersion-based TFR analysis whose tilling is determined with respect to the dispersion

5 curves extracted from the TFR ridges [25]. However, due to the limited information recorded by the single-

6 channel measurement, the extraction of the dispersion characteristics of low-amplitude modes remains

7 challenging, especially for the accurate evaluation of complex medium, such as human long cortical bones

$8[19,29]$.

9 Improvement of the separation of multiple propagation modes superimposed and interfered in the time domain can be achieved using the multichannel recording method combined with some appropriate multichannel data processing techniques $[2-4,20]$. Among them, the most straightforward approach is to map the data from time-distance to the frequency-wavenumber space using the spatio-temporal twodimensional Fourier transform (2-D FT) $[1,2,10]$. In practice, whereas the relatively long duration of the recorded time signals ensures a high frequency resolution, the limited number of positions where these signals are recorded with a finite receiving aperture still results in a low resolution on the wavenumber axis.

Recently, Harley et al. [40] applied compressed sensing to process single-emitter multi-receiver ultrasonic signals for sparse wavenumber extraction of Lamb modes. Actually, since the 1980s, the sparse inversion techniques have been developed in seismic data analysis to overcome the consequence of limited aperture and discretization and to improve the wavenumber resolution [30]. In 1985, Thorson and Claerbout [31] originally proposed the least-squares stochastic inversion, which allows better noise filtering and velocity 
1 and offset space reconstruction in the Radon domain. Sacchi and Ulrych [32] improved the method with an

2 appealing alternative solution based on sparse inversion, called high-resolution Radon transform, which

3 has been generally used for seismic data processing. The high resolution Radon solution employed the non-

4 quadratic regularization constraint, e.g. $l_{1}$-norm, and Cauchy norm, can improve the extraction of

5 dispersion curves compared to Fourier transform. In 2008, Luo et al. [6] applied the high-resolution Radon

6 transform to achieve the sparse representation of the dispersion characteristics of Rayleigh waves. In a

7 continuous effort to improve the resolution of the extracted dispersion trajectories of guided waves in long

8 bone, the high resolution Radon transform has recently been introduced to bone community by Tran et al.

9 [15], which brought to our attention the use of optimization strategies to improve the SVD-based method.

10 Since amplitude and signal-to-noise-ratio (SNR) vary from one mode to another, measurability of modes

is variable and the single-emitter multi-receiver measurement configuration may not be optimal to retrieve all dispersion curves. In order to improve the extraction of the dispersion curves, especially for the poorly detected guided modes, a multi-emitter and multi-receiver transducer array has been developed in our group [19] together with an effective singular vector decomposition (SVD) based signal processing method [19, 33]. The principle of such a multi-emitter and multi-receiver approach has been illustrated on isotropic or transversely isotropic non-dissipative and dissipative materials, including copper plates [19], polymethylacrylate and artificial composite bones [34]. Recently, it has also been applied to data acquired ex vivo on human long cortical bone specimens [16]. However, (i) bone is a highly absorbing material and (ii) measurements are performed using a probe with a relatively small number of receivers [16], which results in a limited SNR and limited resolution of the dispersion curves [15]. 
1 In the present study, we propose a sparse SVD (S-SVD) method, which combines the advantages of

2 SVD and sparse solution to achieve high-resolution extraction of the guided dispersion curves. Different

3 penalty constraints, i.e., $l_{1}$-norm, Frobenius norm and revised Cauchy norm, are compared. The sparse

4 effectiveness and wavenumber resolution are discussed by processing wideband dispersion synthetic

5 signals corrupted with additive Gaussian noise. Finally, the performance of the proposed method is testified

6 using experimental data obtained from a bone-mimicking phantom and from an ex-vivo human radius.

\section{THEORY AND METHODS}

\section{A. Ultrasonic Lamb waves dispersion}

9 Despite the evidence that the geometry of cortical bone is closer to cylindrical shape than to flat plate, there is no clear evidence that tube dispersion curves bring insight in experimentally measured dispersion curves additionally to the plate model [35]. The plate model has already been reported in a few publications to fit experimental data acquired in axial transmission on tubular phantoms [36], bovine bone [37] and 13 human radius [16]. For the working frequency-thickness product $(f \cdot h)$ range between $0.2 \mathrm{MHz} \cdot \mathrm{mm}$ and $142 \mathrm{MHz} \cdot \mathrm{mm}$, the theoretical dispersion curves derived from the plate model were found to be in a good agreement with the experimental data observed in tubular bone-mimicking phantoms [36]. Furthermore, reasonable estimates of mechanical properties and cortical thickness of long bones were reported in two ex-vivo studies using a plate model in the inversion process $[16,37]$. These results suggest that the plate model represents a reasonable approximation for the data measured in cortical bone in the $f \cdot h$ range between $0.2 \mathrm{MHz} \cdot \mathrm{mm}$ and $2 \mathrm{MHz} \cdot \mathrm{mm}$. For lower $f \cdot h$ values (or low frequency excitation) not considered 
1 in the present study, the tube model might be more accurate to fit the experimental dispersion spectra [38].

2 In the present study, the dispersion curves derived from the plate model are considered.

3 According to the vibration pattern, the Lamb modes in isotropic free plates are usually classified as 4 symmetric and anti-symmetric modes following the Rayleigh-Lamb equations [39]. The dispersion curves 5 can be expressed as wavenumber $k$ versus frequency $f=\omega / 2 \pi$ or frequency-thickness product $f \cdot h$.

6 Note that similar dispersion equation can be obtained for absorbing [34] and transversely isotropic free 7 plates [16]. The 2-D FT provides a general relationship between the time and distance space $(x, t)$ and $8 \quad$ wavenumber and frequency space $(k, f)[1,10]$

$$
G(k, f)=\int_{-\infty}^{+\infty} g(x, t) e^{-j(2 \pi f t-k x)} d x d t
$$

where $g(x, t)$ is the signal matrix recorded at a series of different distances $x$. For the ultrasonic Lamb signals, the mode trajectories of the energy distribution in $(k, f)$ domain are in accordance to the dispersion curves, i.e., $k(f)$ obtained using Rayleigh-Lamb equations.

From the signal processing point of view, with a given dispersion curve and an excitation signal, spectrum of the dispersive signal at any propagation distance $x$ can be computed by multiplying a phasespectrum adjustment term $e^{j k(f) x}$ to the spectrum of an excitation. For each mode, the excitation signal is synthesized with a Gaussian spectrum, whose center frequency and bandwidth are selected according to the corresponding $(k, f)$ range of interest. The temporal waveforms can thus be obtained by performing the inverse Fourier transform of the phase-shifted spectrum of excitation. Such a procedure actually provides us an efficient way to synthesize the temporal signal $g(x, t)$ for simulation analysis [26]. 


\section{B. Extraction of the dispersion curves of guided waves}

The problem of obtaining the dispersion curves can be expressed as the accurate estimation of the wavenumbers from the signal matrix $g(t, x)$. Due to the sparsity of the dispersion curves in the $(k, f)$ space, (sparsity means that at each frequency, there only exist a limited number of guided modes with a limited number of loci on wavenumber axis), the basic idea of S-SVD approach is to optimize the data fitting to the experimental observation with a sparse mode energy distribution in the $(k, f)$ space $[32,40]$. Before introducing the S-SVD strategy, we briefly explain the SVD-based extraction of the dispersion curves and the least-squares SVD (LS-SVD)-based extraction of the dispersion curves with an inverse scheme.

\section{(1). SVD-based extraction of the dispersion curves}

Assuming that $M(R, E, t)$ is the three-dimensional (3-D) measurement matrix obtained using the multi-emitter (E) and multi-receiver ( $\mathrm{R}$ ) probe, the modes dispersion relationships can be determined by computing the 2-D FT of $M(R, E, t)$ on $R$ and $t$ axis, hereafter designated by $W(k, E, f)[10,41]$. At each frequency point $f_{p} \in f\left(1,2, \ldots, N_{f}\right)$, The SVD decomposition applied to each response matrix $W\left(k, E, f_{p}\right)$ can be written as [19]

$$
W\left(k, E, f_{p}\right)=U S V^{H}
$$

where $U$ and $V$ are $N_{k} \times N_{k}$ and $N_{E} \times N_{E}$ unitary matrices defining the orthogonal basis in the wavenumber and emitter domains, respectively. ()$^{H}$ is the Hermitian complex conjugate transpose of the matrix. $N_{E}$ and $N_{k}$ are the number of emitters and number of points on the wavenumber axis, respectively.

The diagonal entries of the $N_{E} \times N_{k}$ rectangular matrix $S$ are known as the singular values of $W\left(k, E, f_{p}\right)$. Sparse SVD Method for High Resolution Extraction of the Dispersion Curves of Ultrasonic Guided Waves 8 
1 The columns of $U$ form a set of $N_{k}$ orthogonal vectors, i.e., the $N_{k}$ singular vectors. Each singular vector

2 can be regarded as a function of $k$, which indicates the dispersion information on $k$-axis at a given 3 frequency $f_{p}$.

4 The strategy of the SVD-based noise reduction technique is to discard those small singular values and 5 the corresponding singular vectors which mainly represent noise [19]. Here, the noise is the unwanted 6 signal energy that disturbs the dispersive information estimation, such as the electronic noise. The noise 7 level can be experimentally determined by computing the ratio between the signal amplitude measured 8 before the guided waves arrival and the maximum of the guided wave signal.

At each point $\left(k_{q}, f_{p}\right)$, if only the $N_{r}\left(f_{p}\right)$ first singular vectors are retained instead of the complete $N_{k}$ singular vectors, then the so-called Norm function of the dispersion trajectories is defined as

$$
\operatorname{Norm}\left(k_{q}, f_{p}\right)=\sum_{j=1}^{N_{r}\left(f_{p}\right)}\left|U_{j}\left(k_{q}\right)\right|^{2}
$$

where the scalar $U_{j}\left(k_{q}\right)$ is $q^{\text {th }}$ element of the $j^{t h}$ singular vector $U_{j}$ and the notation $|\cdot|$ corresponds to the

absolute value or modulus of a scalar. Considering the $N_{f}$ frequencies and $N_{k}$ wavenumbers, the dispersion trajectory distribution is obtained as an $N_{k} \times N_{f}$ matrix $\operatorname{Norm}(k, f)$.

Due to the normalized characteristics of the orthogonal basis, the values of Norm function range from 0 to 1 . This value can be interpreted as follows: if a guided mode exists in the signal, the corresponding Norm function value is close to 1 ; otherwise, the value is close to 0 [19]. Note that here the SVD is applied after 2-D FT, in contrast with our previous publication [19] in which the SVD was applied between the temporal and the spatial Fourier transforms. Both methods are theoretically equivalent and lead to the same Norm function. 
However, there are still two limits of such a direct singular value filtering-based method. First, SVD-

2 based method cannot overcome the finite aperture problem [15, 32, 34], which means that the wavenumber

3 0

resolution of the SVD results is identical to that of the 2-D FT results, so that identification of highly overlapping peaks on the wavenumber axis remains challenging. On the other hand, the classical SVDbased strategy, by adjusting the filtering threshold of the singular values, may fail to separate the weak modes from the noise, particularly for modes whose amplitude is close to the noise amplitude. The leastsquares SVD method and S-SVD method may provide new solutions to improve noise filtering and to enhance wavenumber resolution.

\section{(2). Least-squares SVD (LS-SVD)-based extraction of the dispersion curves}

The noise suppression achieved by SVD-based method is fulfilled in the $(k, E)$ domain. Similarly, to further suppress the additive noise on the wavenumber axis, at each frequency point $f_{p}$, Eq. (2) is modified to account for an additive noise in the $(k, E)$ domain, i.e., $N_{k} \times N_{E}$ matrix $n(k, E)$, as follows [42],

$$
S V^{H}=U_{R}^{-1} W\left(k, E, f_{p}\right)+n(k, E)
$$

where $N_{k} \times N_{k}$ matrix $U_{R}=\left[\begin{array}{lllll}U_{1} & U_{2} & \cdots & U_{N r} & 0\end{array}\right]$ which only keeps the $N_{r}\left(f_{p}\right)$ first singular vectors of $U$ associated to the $N_{r}\left(f_{p}\right)$ highest singular values. $U_{R}{ }^{-1}$ is the pseudo-inverse matrix of $U_{R}$. This model aims to accumulating the similar wavenumber characteristics and simultaneously removing the uncorrelated information, i.e., noise, by individual measurement provided by different emitters.

The LS-SVD solution of wavenumber dispersion can be solved by minimizing the following cost function,

$$
J=\left\|U_{R}{ }^{-1} W\left(k, E, f_{p}\right)-S V^{H}\right\|_{F}^{2}+\mu R
$$


$1 \quad\|\cdot\|_{F}^{2}$ represents Frobenius norm of the matrix. $R$ is a penalty term. The Lagrange multiplier $\mu$, also named

2 regularization factor, determines the trade-off between the least-squares fit and the penalty. The 3 wavenumber information is actually contained in both response matrix $W$ and wavenumber basis $U$, so that 4 the cost function is designed with two terms of $U_{R}{ }^{-1} W\left(k, E, f_{p}\right)$ and $S V^{H}$.

5 Considering that the penalty term should be able to suppress the noise existed in the $W\left(k, E, f_{p}\right)$, the 6 quadratic norm $W\left(k, E, f_{p}\right)$, i.e., the Frobenius norm $R_{1}=\left\|W\left(k, E, f_{p}\right)\right\|_{F}^{2}$, is chosen for the penalty term. 7 Substituting $R_{1}$ into Eq. (5), we obtain the solution in the sense of quadratic norm penalty of $W\left(k, E, f_{p}\right)$ 8 by

$$
\nabla J / \nabla W=\left(U_{R}^{-1}\right)^{H} U_{R}^{-1} W-\left(U_{R}^{-1}\right)^{H} S V^{H}+\mu W=0
$$

Thus the LS-SVD solution of Eq. (6) is

$$
\widetilde{W}\left(k, E, f_{p}\right)=\left[\left(U_{R}{ }^{-1}\right)^{H} U_{R}{ }^{-1}+\mu I\right]^{-1}\left(U_{R}{ }^{-1}\right)^{H} S V^{H}
$$

where I denotes the identity matrix. Comparing Eq. (7) with Eq. (2), we actually obtain the least-squares solution of $U$ as

$$
\widetilde{U}=\left[\left(U_{R}^{-1}\right)^{H} U_{R}^{-1}+\mu I\right]^{-1}\left(U_{R}^{-1}\right)^{H}
$$

If $U_{R}$ is the complete orthogonal basis, then $U_{R}{ }^{-1}\left(U_{R}{ }^{-1}\right)^{H}=I$. Eq. (8) is useful, only when $U_{R}{ }^{-1}$ is not a complete orthogonal basis, i.e., the modified $U_{R}$ which only consists of the $N_{r}$ singular vectors. However, without sparse constraints, such a damped least-squares solution is quite limited, which cannot achieve a high resolution [32]. The optimization method for the regularization factor $\mu$ will be discussed later with the sparse strategy. 


\section{(3). High-resolution sparse SVD based extraction of the dispersion curves}

The LS-SVD method describes the optimization scheme that could filter the additive noise. However, in many situations, we may wish to reconstruct a high-resolution sparse result consisting of a few non-zero wavenumber values with the minimal misfit to the experiments. A common approach for obtaining the high-resolution solution is to modify the cost function using the non-quadratic penalty terms. In seismic signal processing, two typical non-quadratic penalty terms, e.g. $l_{1}$-norm and Cauchy norm, have been adapted for the high resolution Radon transform [15, 32, 43-45]. As the sparse characteristics of the guided waves dispersion are on the wavenumber axis, the sparse penalty term should also be designed in $(k, E)$ domain. The $l_{1}$-norm and Cauchy norm for the 2-D matrix $W\left(k, E, f_{p}\right)$ can be defined as

$$
\begin{array}{rlrl} 
& l_{1} \text {-norm: } & R_{2}=\sum_{i=1}^{N_{k}} \sum_{j=1}^{N_{E}}\left|W\left(i, j, f_{p}\right)\right| \\
\text { Cauchy norm: } & R_{3}=\sum_{i=1}^{N_{k}} \ln \left(1+\frac{\sum_{j=1}^{N_{E}}\left|W\left(i, j, f_{p}\right)\right|^{2}}{\sigma^{2}}\right)
\end{array}
$$

where $\sigma$ is the scale factor of the Cauchy distribution. Substituting the $l_{1}$-norm and the Cauchy norm into Eq. (5), the analytical solution cannot be obtained as with LS-SVD method anymore. Some optimization schemes can be considered, for example using the conjugate gradient technique with the forward and adjoint operators [46]. We use the reweighting strategy introduced by Sacchi [43] and also used by Tran $e t$ $a l$. for bone signal processing $[15,44,45]$.

At each frequency point $f_{p}$, the iterative reweighting steps in applying S-SVD are as follows,

1) LS-SVD based initialization of $\widetilde{U}^{(0)}$ and $\widetilde{W}^{(0)}$ according to Eqs. (7-8); 
12 2) For the $n^{\text {th }}$ iteration, computing the $N_{k} \times N_{k}$ reweighting matrix $Q$ using the $l_{1}$-norm and Cauchy 2 norm

$3 \quad l_{1}$-norm:

$$
Q^{\prime}=\left(\begin{array}{ccc}
{\left[\sum_{j=1}^{N_{E}}\left(\left|\widetilde{W}^{(n)}\left(1, j, f_{p}\right)\right|+\sigma^{2}\right)\right]^{-1}} & \ldots & 0 \\
\ldots & \ddots & \ldots \\
0 & \ldots & {\left[\sum_{j=1}^{N_{E}}\left(\left|\widetilde{W}^{(n)}\left(N_{k}, j, f_{p}\right)\right|+\sigma^{2}\right)\right]^{-1}}
\end{array}\right)
$$

5 Cauchy norm:

$$
Q^{\prime \prime}=\left(\begin{array}{ccc}
{\left[\sum_{j=1}^{N_{E}}\left(\left|\widetilde{W}^{(n)}\left(1, j, f_{p}\right)\right|^{2}+\sigma^{2}\right)\right]^{-1}} & & 0 \\
\cdots & \cdots & \cdots \\
0 & \ddots & \\
& \cdots & {\left[\sum_{j=1}^{N_{E}}\left(\left|\widetilde{W}^{(n)}\left(N_{k}, j, f_{p}\right)\right|^{2}+\sigma^{2}\right)\right]^{-1}}
\end{array}\right)
$$

3) Updating the estimated Norm function,

$$
\begin{aligned}
& \widetilde{U}^{(n+1)}=\left[\left(\widetilde{U}^{(n)^{-1}}\right)^{H} \widetilde{U}^{(n)^{-1}}+\mu Q\right]^{-1}\left(\widetilde{U}^{(n)^{-1}}\right)^{H} \\
& \widetilde{W}^{(n+1)}\left(k, E, f_{p}\right)=\widetilde{U}^{(n+1)} S V^{H}
\end{aligned}
$$

where the $n$ is the iteration number.

4) Iteratively solve Eq. (5) by repeating steps (2) and (3); 
1 After the iterative reweighting step, the $\widetilde{U}$ presents the sparse characteristics on the wavenumber axis.

2 Similar to Eq. (3), the sparse wavenumber estimation can thus be obtained by summing the $N_{r}$ first vectors

3 in $\widetilde{U}$. Details of the algorithm can be learned from the appendix. The characteristics of the hyperparameter

$4 \sigma$ will be discussed in Section IV (2).

\section{EXPERIMENTS SETUP}

6 Experiments were performed using an array transducer (Vermon, Tours, France) consisting of 5

7 emitters and 24 receivers associated with a specific electronic device (Althaïs, Tours, France). The pitch of

8 the element is $0.8 \mathrm{~mm}$. The central frequency is $1 \mathrm{MHz}$ and the $-6 \mathrm{~dB}$ frequency bandwidth is from 0.5 to

$9 \quad 1.6 \mathrm{MHz}$. An ultrasound gel (Aquasonic, Parker Labs, Inc, Fairfield, NJ) is used to ensure the coupling between the probe and the measured specimen.

A bone-mimicking plate (Sawbones, Pacific Research Laboratory Inc, Vashon, WA) was first used to record experimental signals. The bone-mimicking material is a transversely isotropic composite made of short glass fibers embedded in an epoxy matrix. One human radius was also tested ex vivo. For result comparisons, the theoretical Lamb modes dispersion curves were computed using a transversely isotropic free plate model [16].

Values of mass density, shear and longitudinal velocities, and thickness utilized to compute the theoretical dispersion curves are listed in table I for both the bone-mimicking material and the human bone. For the bone specimen, we used representative values derived from literature, while for the bone-mimicking plate the values are derived from a previous report by our group [16]. $C_{T}$ is the shear velocity, and $C_{L \|}$, and $C_{L \perp}$ are the compression bulk wave velocities in the directions parallel and normal to the fiber-direction, Sparse SVD Method for High Resolution Extraction of the Dispersion Curves of Ultrasonic Guided Waves 14 
1 respectively. The mass density and thickness are denoted by $\rho$ and $T h$. The average thickness of the human

2 radius specimen was obtained by X-ray high resolution peripheral computed tomography (XtremCT, 3 Scanco Medical, Bruttisellen, Switzerland) [16].

Table I. Values of velocity, density, and thickness of the specimens in the experiments

\begin{tabular}{c|cccc}
\hline \multicolumn{1}{c|}{ Specimens } & $\rho\left(\mathrm{g} \cdot \mathrm{cm}^{-3}\right)$ & $T h(\mathrm{~mm})$ & $C_{T}\left(\mathrm{~mm} \cdot \mu \mathrm{s}^{-1}\right)$ & $\left(C_{L \|}, C_{L \perp}\right)\left(\mathrm{mm} \cdot \mu \mathrm{s}^{-1}\right)$ \\
\hline Bone-mimicking Plate & 1.64 & 4 & 1.62 & $\left(C_{L \|}, C_{L \perp}\right)=(3.57,2.91)$ \\
Human Radius Specimen & 1.85 & 2.50 & 1.8 & $\left(C_{L \|}, C_{L \perp}\right)=(4.0,3.41)$ \\
\hline
\end{tabular}

\section{RESULTS}

A. Synthetic wideband signals

(1). 2-D FT and SVD results

The method was first assessed on synthetic signals representing an idealized experiment on a $2 \mathrm{~mm}$ -

thick bone-mimicking plate with our array transducer. As shown in Fig. 1a, the 24 channel synthetic wideband signals excited by first emitter are plotted in a time-distance (r-t) diagram. Signals corresponding to six fundamental wide $k$-band $(0<k<4 \mathrm{rad} / \mathrm{mm})$ Lamb modes A0, S0, A1, S1, A2 and S2 were synthesized according to [26], with peak-to-peak amplitudes of $1,0.3,1,0.3,1$, and 0.3 , respectively. A Gaussian noise was added into each channel of the signal array with a fixed SNR of $15 \mathrm{~dB}$. The 2-D FT result of the received signal after the first emission is presented in Fig. 1b, where the low-amplitude modes (S0, S1 and S2) are too low to be identified. Fig. 1c depicts the first partial Norm function obtained using only the first singular vector $U_{1}$ (corresponding to the highest singular value) at each frequency. Fig. 1d shows the second partial Norm function corresponding to the second singular vectors $U_{2}$. Fig. 1e shows the Norm function calculated 
1 according to Eq. (3) by summing these two partial functions, i.e., $\left|U_{1}\right|^{2}+\left|U_{2}\right|^{2}$. Fig. 1f compares the

2 partial Norm functions with the wavenumber spectrum obtained using 2-D FT method at a fixed frequency

3 of $0.5 \mathrm{MHz}$. This particular frequency is indicated by red dot vertical lines in Figs. 1(b-e). The $\left|U_{1}\right|^{2}$ and

$4 \quad\left|U_{2}\right|^{2}$ are shown with symbols $(\triangle$ and $\diamond)$, respectively. The Norm function reconstructed by summing

5 amplitude-squares of two first singular vectors, i.e., $\left|U_{1}\right|^{2}+\left|U_{2}\right|^{2}$, is shown in red solid line. The

6 normalized 2-D FT results of the first emission is shown with black dash line. Compared with the 2D-FT

7 method, the SVD-based method enables to detect the dispersion trajectories for the weak modes. For

8 example, the low-amplitude S0 mode obtained by 2D-FT (see Fig. 1b) is significantly enhanced by using

9 SVD-based method (see Figs. 1e), which can also be confirmed in Fig. 1f by comparing the dash and solid lines obtained by using two different methods. 

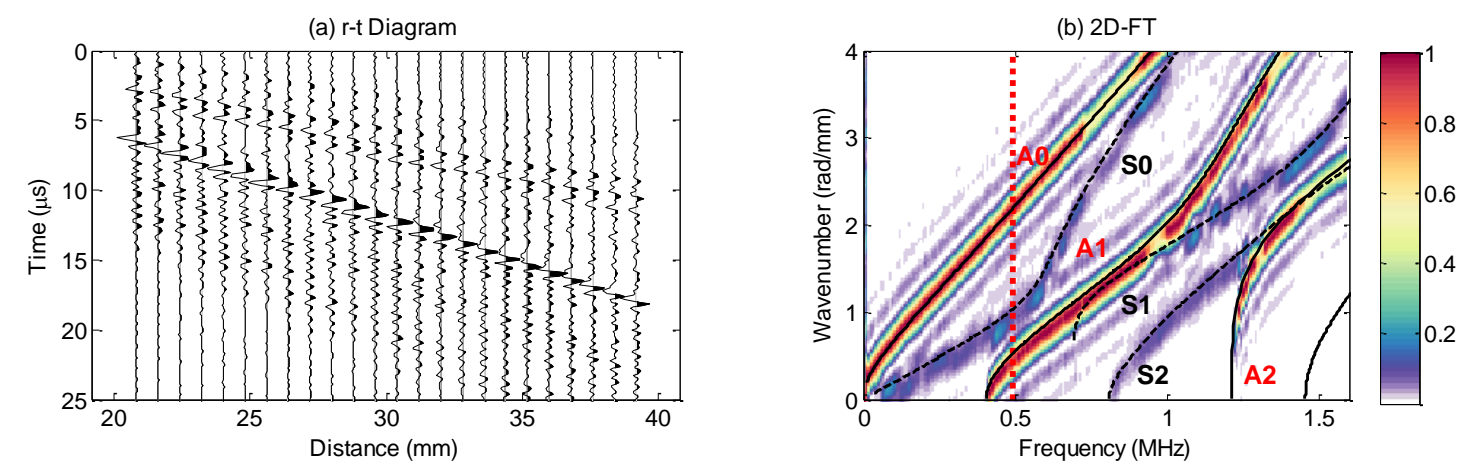

(c) SVD, $\left|U_{1}\right|^{2}$
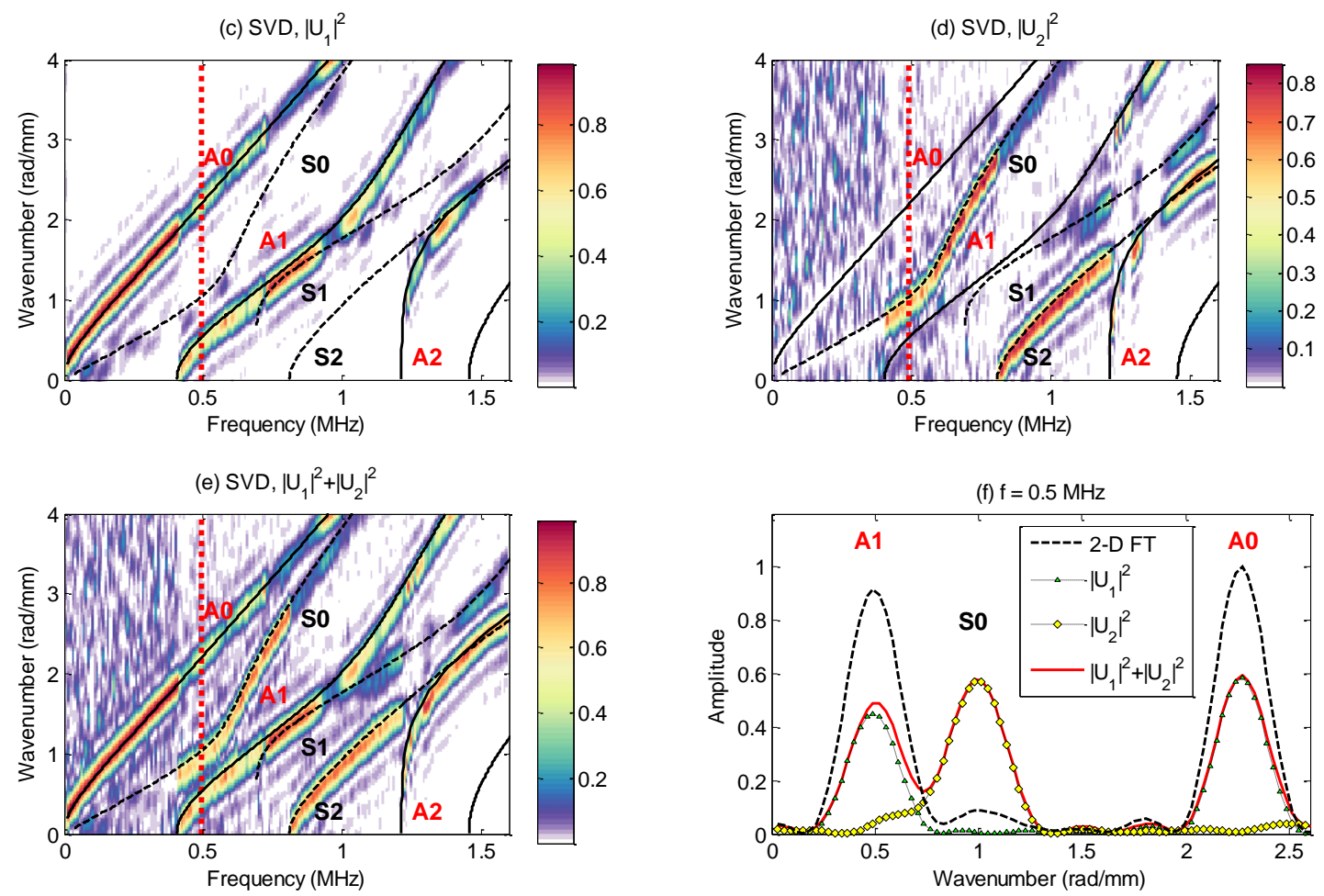

2 Fig. 1. The synthetic signals, (a) distance-time (r-t) diagram, (b) dispersion energy in $(k, f)$ space using 2-D FT method,

3 (c) first partial Norm function obtained with the first singular vectors $\left|U_{1}\right|^{2}$ corresponding to the highest singular values

4 for all frequencies, (d) second partial Norm function of second singular vectors $\left|U_{2}\right|^{2}$ for all frequencies, (e) Norm function

5 obtained by summing two first singular vectors $\left|U_{1}\right|^{2}$ and $\left|U_{2}\right|^{2}$, i.e., $\left|U_{1}\right|^{2}+\left|U_{2}\right|^{2}$, for all frequencies, (f) comparison of

6 the wavenumber functions obtained using SVD-based method and 2-D FT method at $0.5 \mathrm{MHz}$. Please note that the $|\cdot|^{2}$ is

7 the amplitude-square of each element of the matrix. 
Optimization of the factor $\mu$ and hyperparameter $\sigma$ ensures the fidelity and the stability of the 3 regularization penalty. Fig. 2 shows the $\left\|W\left(k, E, f_{p}\right)\right\|_{F}^{2}$ curves of the multimodal signals in Fig. 1 versus 4 common $\operatorname{logarithm} \log _{10}(\mu)$ for the frequency fixed at $1 \mathrm{MHz}$. It can be found that the $\left\|W\left(k, E, f_{p}\right)\right\|_{F}^{2}$ 5 curve based on the LS-SVD method shows a constant norm without sparse property, whereas the $6\left\|W\left(k, E, f_{p}\right)\right\|_{F}^{2}$ results of S-SVD present a left-right-flipped-Z-shape trend with two turning points. The 7 first turning points of S-SVD $\left(l_{1}\right.$-norm) and S-SVD (Cauchy norm) on the $\left\|W\left(k, E, f_{p}\right)\right\|_{F}^{2}$ curves occur 8 around $\mu=10^{1.5}$ and $10^{-1.5}$, respectively, which can achieve the sparse results. The second turning points 9 of S-SVD $\left(l_{1}\right.$-norm) and S-SVD (Cauchy norm) on the $\left\|W\left(k, E, f_{p}\right)\right\|_{F}^{2}$ curves occur at $\mu=10^{6}$ and 10 , 10 respectively, which actually indicates the change between high resolution and no sparsity. High reslution 30 results with different sparse degrees can be obtained in between of the two turning points.

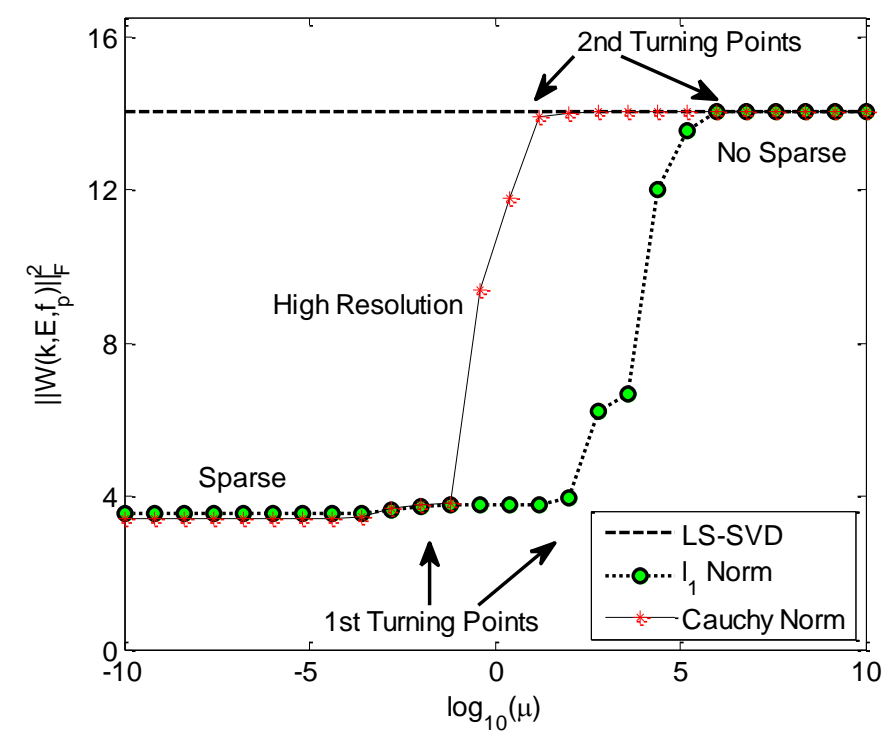

Fig. 2. The $\left\|W\left(k, E, f_{p}\right)\right\|_{F}^{2}$ variation versus $\log _{10}(\mu)$ using different penalty functions, for the multimodal synthetic signals at $1 \mathrm{MHz}$. 
1 According to Eq. (10), if the value of hyperparameter $\sigma$ is too large compared to that of $\left|W\left(k, E, f_{p}\right)\right|$,

2 the reweighting matrix $Q$ will be only determined by the value of $\sigma$. The value of $\sigma$ should be much smaller

3 than the magnitude of $\left|W\left(k, E, f_{p}\right)\right|$. An heuristic value of 0.02 was adopted for $\sigma$ in the present study.

4 Commonly, a relatively small value of regulation parameter $\mu$ leads to solutions with the best fit and

5 insignificant estimation error, while large $\mu$ can enhance the penalty effects with high-resolution sparsity

$6[15,44,45,47]$. However, due to the use of the inverse matrix $U^{-1}$ in our model in Eq. (4), an opposite

7 relationship is observed between the sparsity and the regularization parameter. As shown in Fig. 2, a small

8 value of $\mu$ can guarantee the sparse convergence of the S-SVD method, while the use of a large $\mu$ value

9 actually is corresponding to no sparse results as similar as the LS-SVD method. Furthermore, $\mu=0$ cannot achieve the correct reweighting either. Those statuses between the first and second turning points can be used to tune the sparsity with different resolutions.

\section{(3). LS-SVD and high-resolution sparse SVD results of the synthetic data}

Figure 3 shows the Norm functions obtained by applying SVD, LS-SVD, and S-SVD $\left(l_{1}\right.$-norm and Cauchy norm) to the synthetic signals. Compared with the 2-D FT results (in Fig. 1), the low amplitude modes (S0, S1 and S2) are successfully enhanced by the SVD-based processing in Fig. 3a. As shown in Fig. 3b, there is no improvement of the wavenumber resolution using the LS-SVD method ( also see Fig. 47

4). Figs. 3(c-f) present the S-SVD results with different resolution, two group of different regularization parameters, i.e., $\mu=0.5,1000$ and $\mu=0.01,1$, are used for S-SVD ( $l_{1}$-norm) and S-SVD (Cauchy norm), respectively. It can be observed that the small $\mu$ selected around the first turning point of Fig. 2 yields highsparse estimates of the dispersion curves (Figs. 3c and 3e). Furthermore, choosing the suitable $\mu$ values, 
1 the S-SVD method can converge to different sparse levels (Figs. 3d and 3f) with different resolutions (also

2 see Fig. 4). The convergence characteristics actually change with the frequencies, but Fig. 3 also confirmed

3 that $\mu$ is stable enough to achieve a similar sparse level at different frequencies.

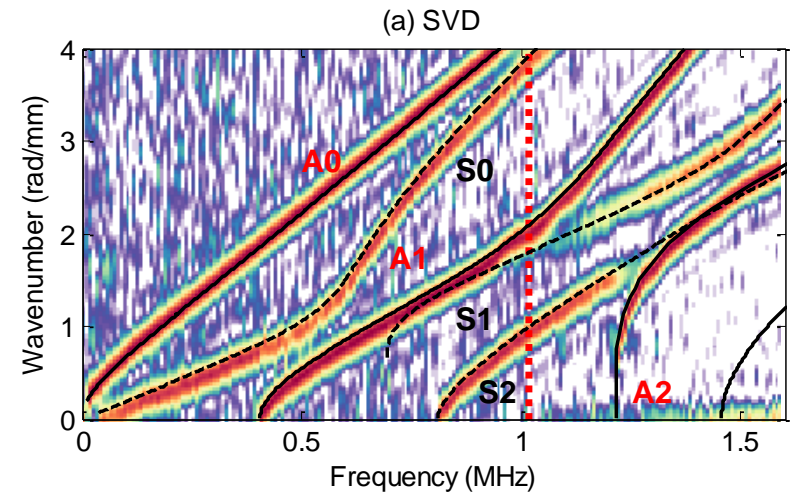

(c) S-SVD $\left(\mathrm{I}_{1}\right),(\mu, \sigma)=(0.5,0.02)$

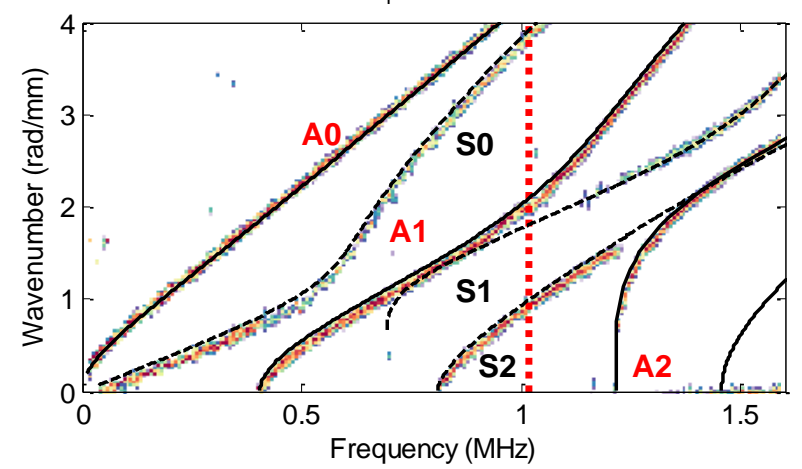

(e) S-SVD (Cauchy), $(\mu, \sigma)=(0.01,0.02)$

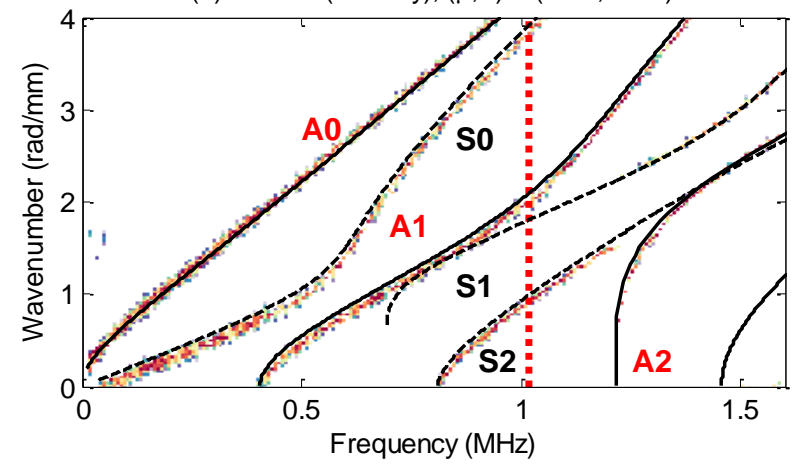

(b) LS-SVD, $(\mu=100)$

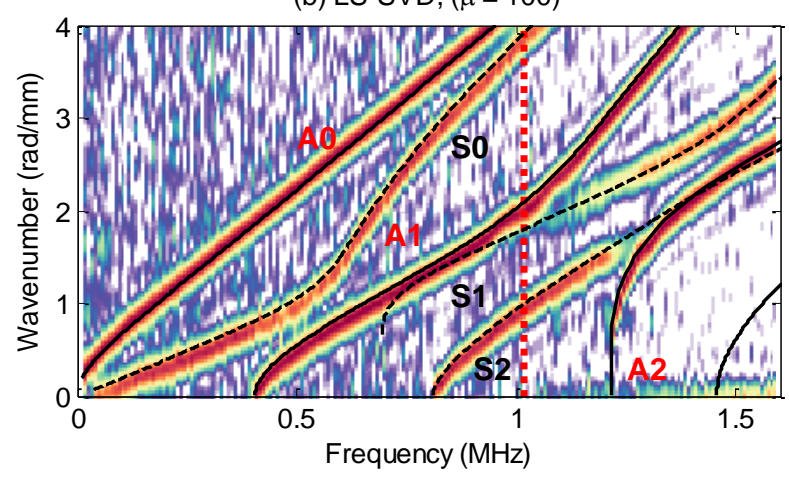

(d) S-SVD $\left(I_{1}\right),(\mu, \sigma)=(1000,0.02)$

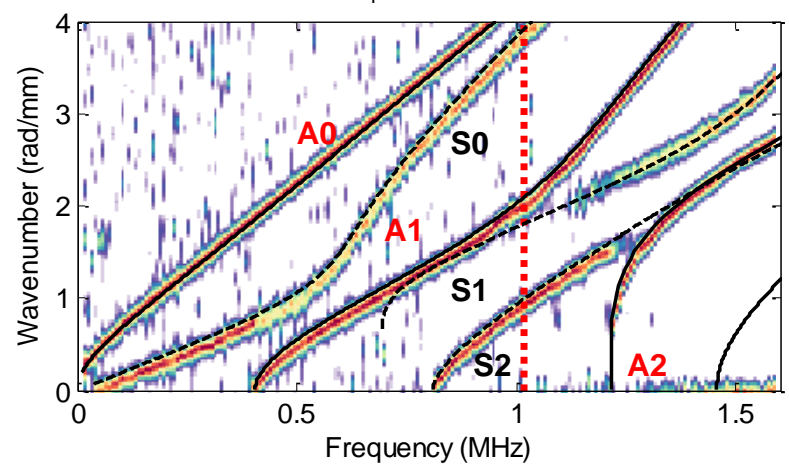

(f) S-SVD (Cauchy), $(\mu, \sigma)=(1,0.02)$

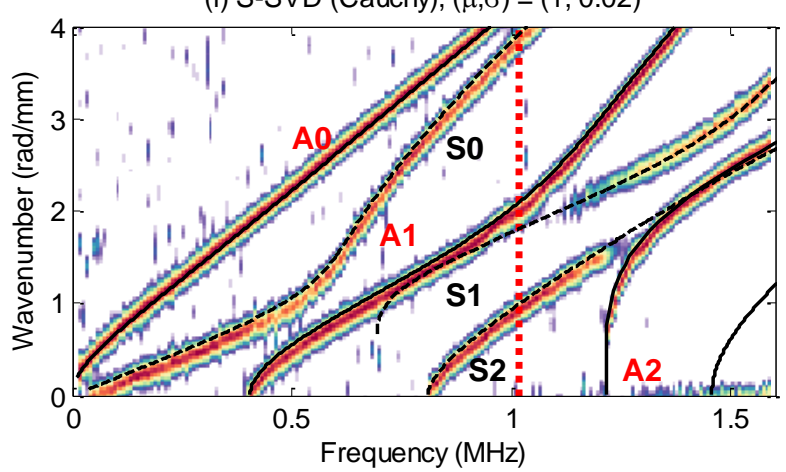
signals corresponding to a $2 \mathrm{~mm}$-thick bone-mimicking plate. 


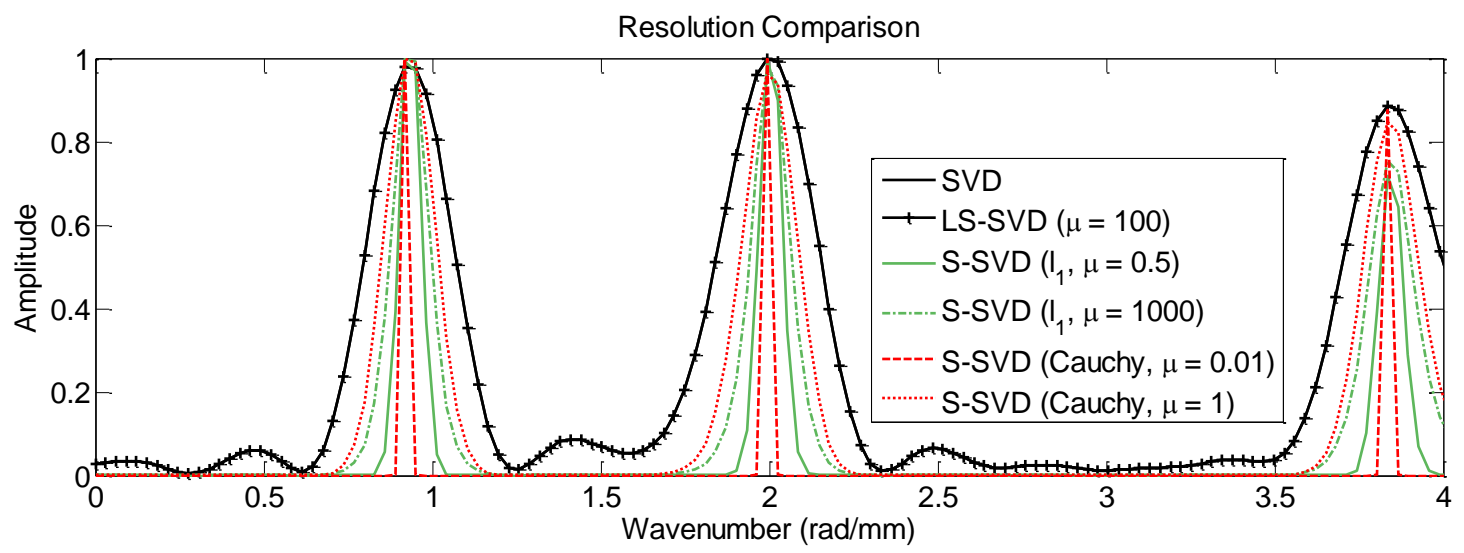

2 Fig. 4. Comparison of wavenumber resolution at $1 \mathrm{MHz}$ of the synthetic signals corresponding to a 2 mm-thick bone-

mimicking plate.

$4 \quad$ Figure 4 compares the Norm function obtained at $1 \mathrm{MHz}$ (indicated as red dot lines in Fig. 3) using

5 different methods and different parameters. The SVD and LS-SVD provide comparable results with the

6 same resolution as that of the 2-D FT. In this study, the wavenumber resolution corresponding to the probe

7 employed can be computed by $2 * 2 \pi /(24 * 0.8)=0.65 \mathrm{rad} / \mathrm{mm}$, where the 24 and $0.8 \mathrm{~mm}$ are number

8 of the elements and pitch size, respectively. For example, the main lobe width of S2 mode, extracted by the

9 SVD and LS-SVD method, is equal to $0.65 \mathrm{rad} / \mathrm{mm}$ locating between 0.54 and $1.19 \mathrm{rad} / \mathrm{mm}$ in Fig. 4. An

10 improved resolution is reached using the S-SVD method. For instance, the main lobe of the S2 mode

extracted from the amplitude curves of S-SVD (Cauchy, $\mu=0.01$ ) has a width of $0.11 \mathrm{rad} / \mathrm{mm}$, i.e., 6 times improvement compared to the classical 2-D FT resolution, approximately. As shown in Fig. 2, such a $\mu$ value is adopted according the first turning point. It also illustrates that using the S-SVD method, only

143 sharp peaks are observed and the mode energy leakage is completely suppressed. However, with such a 15 wavenumber resolution obtained by S-SVD, it is still not high enough to resolve those severely overlapped modes, such as $\mathrm{A} 1$ and $\mathrm{S} 1$ modes in range of 0.7 to $1 \mathrm{rad} / \mathrm{mm}$. 


\section{$1 \quad$ B. Phantom experiment}

2 Figure 5 displays the SVD and S-SVD results of the experiment signal measured in the 4 mm-thick

3 Sawbone plates. The results are also presented in $(k, f)$ domain with the wavenumber dispersion curves.

4 Similarly to the results of synthetic signals, the S-SVD method can improve the extraction of the dispersion

5 curves with sparse energy focusing and noise filtering.

6 The main lobes of the S0 and A1 mode illustrated from the amplitude curves of the S-SVD (Cauchy

7 norm) with $(\mu, \sigma)=(0.0015,0.02)$ and $\operatorname{S-SVD}\left(l_{1}\right.$-norm $)$ with $(\mu, \sigma)=(10,0.02)$ are in widths of 0.28

$8 \mathrm{rad} / \mathrm{mm}$ and $0.18 \mathrm{rad} / \mathrm{mm}$, respectively. In particular, the Norm function extracted at $1 \mathrm{MHz}$ in the range of

91 to $2 \mathrm{rad} / \mathrm{mm}$ shows that the sparse SVD (Cauchy norm) enables to separate two wavenumber peaks, and the sparse SVD $\left(l_{1}\right.$-norm) finds three wavenumber peaks, when the SVD finds a single large peak (see Fig. 5d). Actually, in this wavenumber range, the plate model predicts the presence of two pairs of close modes 12 A2, S2 and A3, S3, respectively. The wavenumber resolution enhancement achieved with S-SVD, in 13 contrast to SVD, allows separation of the two pairs of close modes. The finest resolution of S-SVD $\left(l_{1}\right.$ - 
(a) SVD

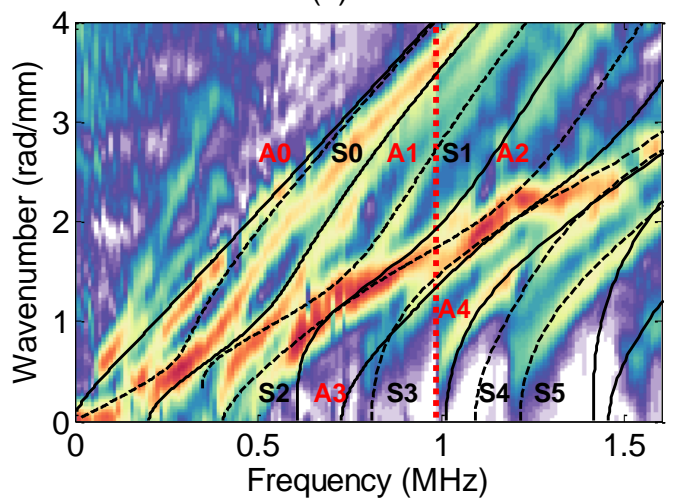

(c) S-SVD(Cauchy), $(\mu, \sigma)=(0.0015,0.02)$

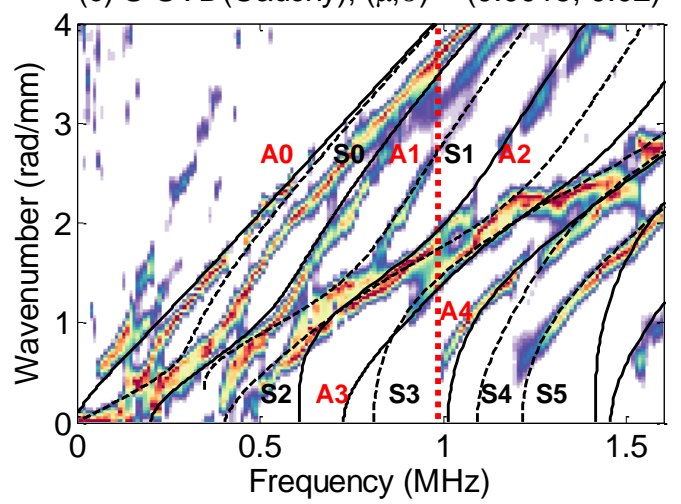

(b) $\operatorname{S-SVD}\left(I_{1}\right),(\mu, \sigma)=(10,0.02)$

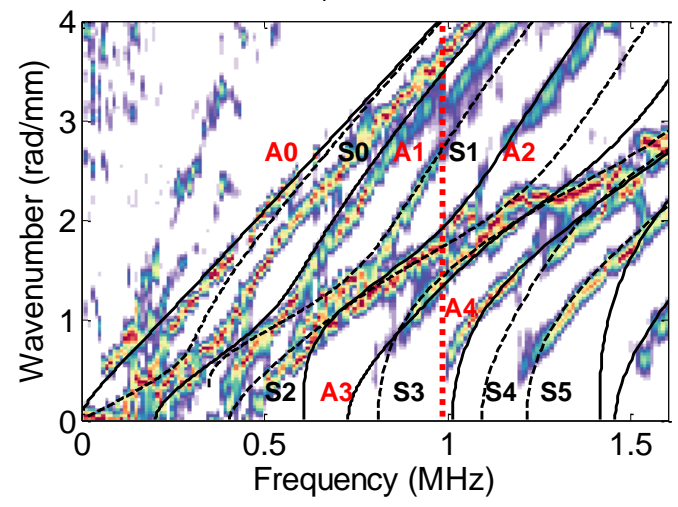

(d) Resolution Comparison

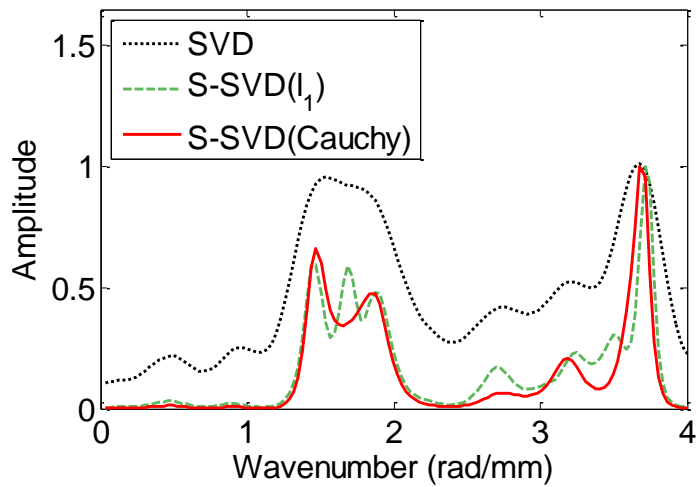

2 Fig. 5. Multimode dispersion curves measured in a 4 mm-thick bone-mimicking plate, SVD and S-SVD $\left(l_{1}\right.$-norm and Cauchy norm) results, comparison of wavenumber resolution at $1 \mathrm{MHz}$.

C. Ex-vivo experiments

Results presented in Fig. 6 refer to the signals measured in a $2.5 \mathrm{~mm}$-thick human radius ex vivo. Due

7 limit in clinical measurement still induces strong mode aliasing, which is challenging for mode

identification. As shown in Figs. $6 \mathrm{~b}$ and $6 \mathrm{c}$, in agreement with the simulation and phantom studies, the S-

9 SVD method can filter most of the noise and yields energy concentrated trajectories. Because at $1 \mathrm{MHz}$, 60

some modes, like S0, S1 and A1 are poorly excited, the amplitude curves at $0.5 \mathrm{MHz}$ are plotted in Fig. $6 \mathrm{~d}$, which also confirms the high wavenumber resolution of the S-SVD method. The main lobes of the A0 Sparse SVD Method for High Resolution Extraction of the Dispersion Curves of Ultrasonic Guided Waves 23 
1 mode illustrated from the amplitude curves of the S-SVD $\left(l_{1}\right.$-norm $)$ with $(\mu, \sigma)=(500,0.02)$ and S-SVD

2 (Cauchy norm) with $(\mu, \sigma)=(0.2,0.02)$ are in widths of $0.22 \mathrm{rad} / \mathrm{mm}$ and $0.20 \mathrm{rad} / \mathrm{mm}$, respectively.

(a) SVD

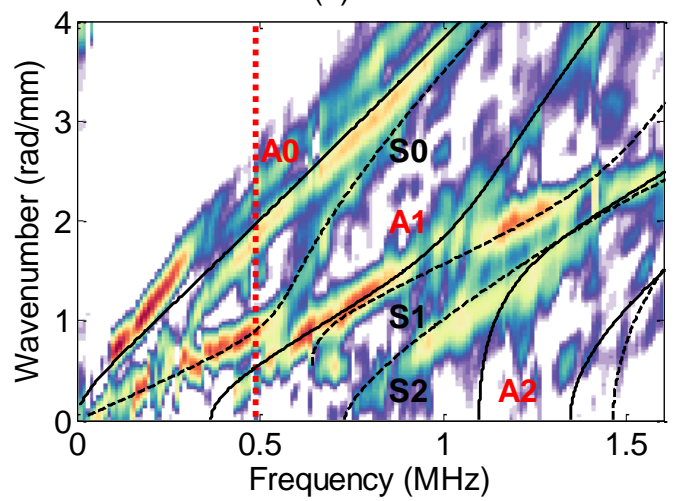

(c) S-SVD(Cauchy), $(\mu, \sigma)=(0.2,0.02)$

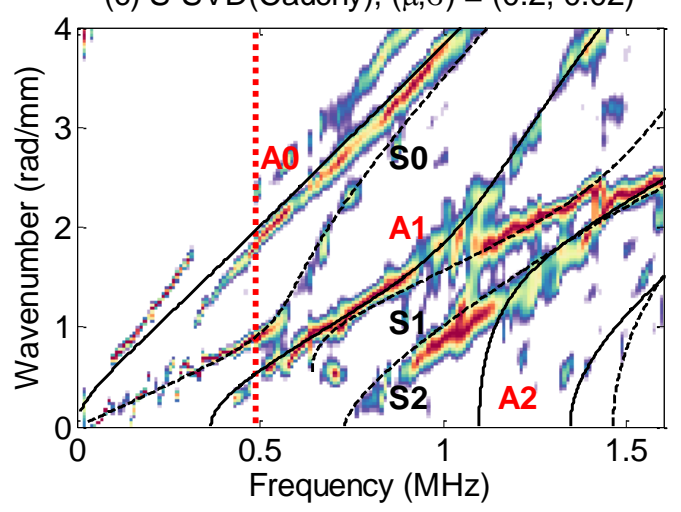

(b) $\operatorname{S-SVD}\left(\mathrm{I}_{1}\right),(\mu, \sigma)=(500,0.02)$

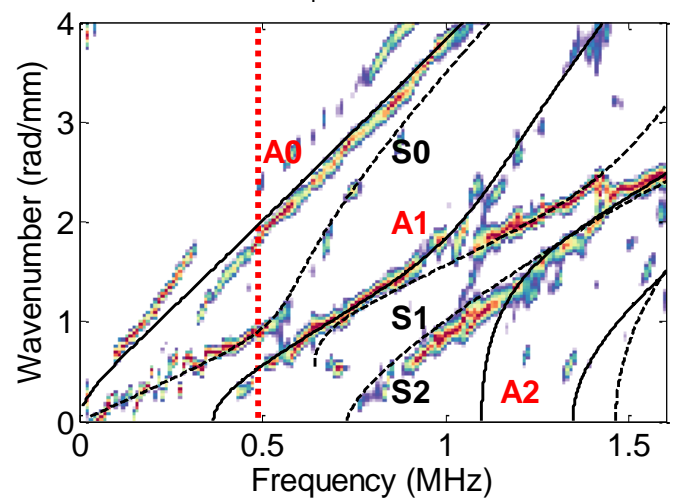

(d) Resolution Comparison

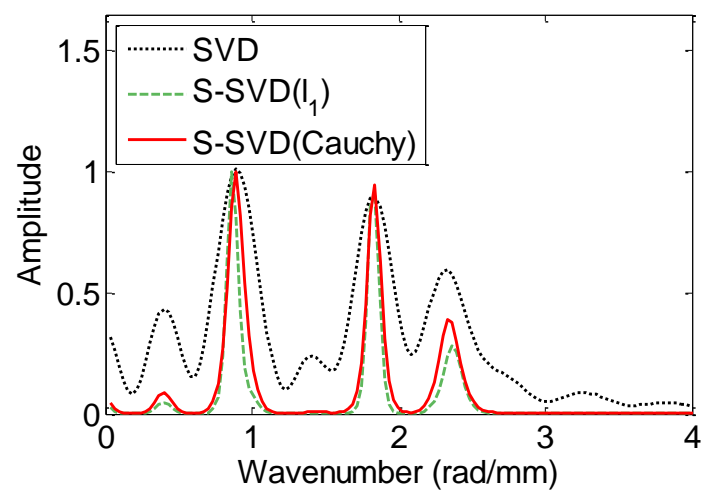

Fig. 6. Multimode dispersion curves measured in a $2.5 \mathrm{~mm}$-thick human radius (ex vivo), SVD and S-SVD $\left(l_{1}\right.$-norm, and Cauchy norm) results, comparison of wavenumber resolution at $0.5 \mathrm{MHz}$.

\section{DISCUSSION}

8
9 measurable length of several centimeters where only tense of transducer elements can actually be arranged.

The limitation is due to accessibility of clinical sites such as forearm. Currently, a SVD-based signal 
1 processing approach allows efficient noise filtering and weak modes amplitude enhancement. However,

2 the relatively poor spatial sampling results in poor resolution on the wavenumber $k$-axis, which still

3 prevents a clear identification of the dispersion curves of overlapping modes. The sparse strategy continues

4 to attract considerable attention with capability of resolution improvement beyond the classical resolution

5 limit. In this paper, a S-SVD method for sparse characterization of the dispersion curves is proposed to

6 overcome the limited wavenumber resolution of ultrasonic guided waves measurement. The method is

7 proposed, assuming that the additional noise, which is weakly correlated to the singular vectors of the SVD

8 decomposition, can be optimally removed to promote a sparse result.

9 In agreement with previous studies $[15,40,44,45]$, our results also illustrate that the choice of the penalty term is important for the sparse optimization. The use of the $l_{2}$-norm penalty in our cost function basically leads to the least-squares solution, without obvious improvement of the wavenumber resolution.

Furthermore, it seems that the proposed LS-SVD method cannot effectively remove the additive noise either. In contrast, the use of $l_{1}$-norm and Cauchy norm can enhance the extraction of the dispersion curves with a high wavenumber resolution, i.e., sparse characteristics [15, 44, 45]. As illustrated in the synthetic and experimental results, both $l_{1}$-norm and Cauchy penalty terms can provide good results with significant resolution enhancement, so that it is difficult to directly conclude which of them can provide the finest Norm function with the best wavenumber resolution. Certainly, other penalty terms are also worth to be investigated, for instance the $l_{p}$-norm or even some polynomial terms (the sum of different norms). The trade-off could be that the complicated design of the cost function will also raise other challenges, such as the optimization of the regularization factors and the convergence criteria. 
1 Regularization schemes are commonly accepted to solve ill-posed problems. The performance of S-

2 SVD method highly relies on the choice of a suitable regularization parameter. Unsuitable regularization

3 will either enforce the over-sparse effectiveness to the all-zero solution or provide an insufficient sparse

4 solution without enough enhancement. We used an heuristic method to optimize the regularization

5 parameter. As shown in Fig. 2, when the regularization parameter increases, the norm $\left\|\widetilde{W}\left(k, E, f_{p}\right)\right\|_{F}^{2}$

6 convergence curves present two turning points with the left-right-flipped-Z-shape trends. The first and

7 second turning points of the $\left\|\widetilde{W}\left(k, E, f_{p}\right)\right\|_{F}^{2}$ curve are on the boundaries of the sparsity, high-resolution

8 and no-sparsity, respectively. The results suggest that the first turning point can be chosen as the suitable

9 value of the regularization parameter with sparsity optimization. Furthermore, the values of the regularization parameter between the two turning points enable to tune the sparse level with different resolutions. A second hyperparameter $\sigma$ is imposed on the penalty function. It can be understood as a small additive perturbation that represents the default power in absence of hyperbolic events [32]. A 1-D search based on Brent parabolic interpolation could be used to optimize $\sigma[43,48]$. However, it should be noted that the convergence characteristics also vary with frequencies. Strictly speaking, the regularization parameter and hyperparameter must be optimized at each frequency. In our study, we find that they are stable enough to allow us to choose identical values for different frequencies. Furthermore, there is also a trade-off between the sparsity/resolution and capability of mode retrieve, since the very high resolution also enhances the over-sparse effectiveness with drawback of removing some of the useful information. To maximize the dispersion information, a suitable sparse level should be chosen with balance between the misfit and resolution, allowing to remaining the weak modes together with sufficient separation of the overlap dispersion curves. Sparse SVD Method for High Resolution Extraction of the Dispersion Curves of Ultrasonic Guided Waves 26 
1 Other signal processing methods, such as the spectrum estimation and high-resolution Radon method $2[15,44,45]$ can also be considered to achieve a high-resolution wavenumber distribution. However, to the 3 best authors' knowledge, currently, most of the proposed methods have been designed for single-emitter 4 multi-receiver processing. In contrast, our SVD-based approach takes advantage of both the multichannel 5 transmission and reception. Specifically, the proposed S-SVD method combines the advantages of the 6 SVD-based enhancement of low-amplitude modes and also of the sparse penalty scheme to filter the noise 7 with high wavenumber resolution. Such a method may be more robust for detecting those weak modes 8 severely corrupted by noise, especially when measuring highly damping materials, such as bone.

9 The S-SVD method developed in the study involves matrix inversion in the SVD iteration with a relatively expensive computation. However, in general, 10 to 20 times iterations are sufficient to converge to the sparse solution, which suggests that the S-SVD method is capable for the real-time multi-emitter and multi-receiver data processing.

\section{CONCLUSION}

This original S-SVD approach discussed in this study combines the SVD-based SNR improvement and the advantage of sparse regularization strategy to successfully achieve high resolution extraction of the dispersion curves of ultrasonic guided waves. The analysis of the synthetic signals and experimental data illustrates that the S-SVD method may provide significant advantages when trying to retrieve the characteristics of the waveguide using model-based inverse procedures for three reasons:

i) The sparse strategy can overcome the practical problem of the finite aperture caused by the limit size and small number of elements of the probe. The S-SVD method allows retrieving Sparse SVD Method for High Resolution Extraction of the Dispersion Curves of Ultrasonic Guided Waves 27 
the dispersion curves with high wavenumber resolution, so that some severely overlapping guided modes can be separated;

ii) The merits of the SVD-based method and the high resolution optimization are preserved, which allows extraction of some weak modes severely corrupted by noise;

iii) The robust convergence characteristics of the regularization parameters allow the convenient implementation of the S-SVD method. Furthermore, the left-right-flipped-Z-shape trend of the sparse Norm function provides a flexible way for tuning the sparsity of the dispersion trajectories for different applications.

The existence of surrounding soft tissues is expected to affect the SNR and signal coherence. Future work will focus on adapting the S-SVD method on processing the in vivo guided waves data.

\section{ACKNOWLEDGMENT}

\section{References:}

[1] C. H. Chapman, "A new method for computing synthetic seismograms," Geophysical Journal International, vol. 54, pp. 481-518, 1978. 
1 [2] G. A. McMechan and M. J. Yedlin, "Analysis of dispersive waves by wave field transformation," Geophysics, vol. 46, pp. $2869-874,1981$.

3 [3] P. Gabriels, R. Snieder and G. Nolet, "In situ measurements of shear-wave velocity in sediments with higher-mode Rayleigh 4 waves," Geophysical prospecting, vol. 35, pp. 187-196, 1987.

5 [4] C. B. Park, R. D. Miller and J. Xia, "Multichannel analysis of surface waves," Geophysics, vol. 64, pp. 800-808, 1999.

[5] G. D. Bensen, M. H. Ritzwoller, M. P. Barmin, A. L. Levshin, F. Lin, M. P. Moschetti, N. M. Shapiro, and Y. Yang, "Processing seismic ambient noise data to obtain reliable broad-band surface wave dispersion measurements," Geophysical Journal International, vol. 169, pp. 1239-1260, 2007.

[6] Y. Luo, J. Xia, R. D. Miller, Y. Xu, J. Liu, and Q. Liu, "Rayleigh-wave dispersive energy imaging using a high-resolution linear Radon transform," Pure and Applied Geophysics, vol. 165, pp. 903-922, 2008.

[7] A. Papandreou-Suppappola, R. L. Murray, B. G. Iem, and G. F. Boudreaux-Bartels, "Group delay shift covariant quadratic time-frequency representations," IEEE Transactions on Signal Processing, vol. 49, pp. 2549-2564, 2001.

[8] G. Le Touze, B. Nicolas, J. I. Mars, and J. L. Lacoume, "Matched representations and filters for guided waves," IEEE Transactions on Signal Processing, vol. 57, pp. 1783-1795, 2009.

[9] J. Bonnel, G. E. G. Le Touz E, B. Nicolas, and J. Mars, IEEE Signal Processing Magazine, vol. 30, pp. 120-129, 2013.

[10] D. Alleyne and P. Cawley, "A two-dimensional Fourier transform method for the measurement of propagating multimode signals," Journal of the Acoustical Society of America, vol. 89, pp. 1159-1168, 1991.

[11] W. H. Prosser, M. D. Seale and B. T. Smith, "Time-frequency analysis of the dispersion of Lamb modes," Journal of the Acoustic Society of America, vol. 105, pp. 2669-2676, 1999.

[12] P. Wilcox, M. Lowe and P. Cawley, "The effect of dispersion on long-range inspection using ultrasonic guided waves," NDT\&E International, vol. 34, pp. 1-9, 2001.

[13] S. Fateri, N. V. Boulgouris, A. Wilkinson, W. Balachandran, and T. Gan, IEEE Transactions on Ultrasonics, Ferroelectrics, and Frequency Control, vol. 61, pp. 1515-1524, 2014.

[14] K. Xu, D. Ta and W. Wang, "Multiridge-based analysis for separating individual modes from multimodal guided wave signals in long bones," IEEE Transactions on Ultrasonics, Ferroelectrics and Frequency Control, vol. 57, pp. 2480-2490, 2010. [15] T. N. Tran, K. T. Nguyen, M. D. Sacchi, and L. H. Le, "Imaging ultrasonic dispersive guided wave energy in long bones using linear Radon transform," Ultrasound in Medicine and Biology, vol. 40, pp. 2715-2727, 2014-11-01 2014.

[16] J. Foiret, J. G. Minonzio, C. Chappard, M. Talmant, and P. Laugier, "Combined estimation of thickness and velocities using ultrasound guided waves: a pioneering study on in vitro cortical bone samples," IEEE Transactions on Ultrasonics, Ferroelectrics and Frequency Control, vol. 61, pp. 1478-1488, 2014.

[17] M. Bernal, I. Nenadic, M. W. Urban, and J. F. Greenleaf, "Material property estimation for tubes and arteries using ultrasound radiation force and analysis of propagating modes," Journal of the Acoustical Society of America, vol. 129, pp. 13441354, 2011.

[18] J. Xia, Y. Xu and R. D. Miller, "Generating an image of dispersive energy by frequency decomposition and slant stacking," Pure and Applied Geophysics, vol. 164, pp. 941-956, 2007.

[19] J. G. Minonzio, M. Talmant and P. Laugier, "Guided wave phase velocity measurement using multi-emitter and multireceiver arrays in the axial transmission configuration," Journal of the Acoustical Society of America, vol. 127, pp. 2913-2919, 2010.

[20] L. Wang, Y. Xu, J. Xia, and Y. Luo, "Effect of near-surface topography on high-frequency Rayleigh-wave propagation," Journal of Applied Geophysics, vol. 116, pp. 93-103, 2015.

[21] L. Cohen, "Time-frequency distributions-a review," Proceedings of the IEEE, vol. 77, pp. 941-981, 1989.

Sparse SVD Method for High Resolution Extraction of the Dispersion Curves of Ultrasonic Guided Waves 29 
[22] L. De Marchi, A. Marzani, S. Caporale, and N. Speciale, "Ultrasonic guided-waves characterization with warped frequency transforms," IEEE Transactions on Ultrasonics, Ferroelectrics and Frequency Control, vol. 56, pp. 2232-2240, 2009.

[23] M. Zhao, L. Zeng, J. Lin, and W. Wu, "Mode identification and extraction of broadband ultrasonic guided waves," Measurement Science and Technology, vol. 25, p. 115005, 2014.

[24] Y. Yang, Z. K. Peng, W. M. Zhang, G. Meng, and Z. Q. Lang, "Dispersion analysis for broadband guided wave using generalized warblet transform," Journal of Sound and Vibration, vol. 367, pp. 22-36, 2016.

[25] J. Hong, K. H. Sun and Y. Y. Kim, "Dispersion-based short-time Fourier transform applied to dispersive wave analysis," Journal of the Acoustical Society of America, vol. 117, pp. 2949-2960, 2005.

[26] K. Xu, D. Ta, P. Moilanen, and W. Wang, "Mode separation of Lamb waves based on dispersion compensation method," Journal of the Acoustical Society of America, vol. 131, pp. 2714-2722, 2012.

[27] R. Sicard, J. Goyette and D. Zellouf, "A numerical dispersion compensation technique for time recompression of Lamb wave signals," Ultrasonics, vol. 40, pp. 727-732, 2002.

[28] P. D. Wilcox, "A rapid signal processing technique to remove the effect of dispersion from guided wave signals," IEEE Transactions on Ultrasonics, Ferroelectrics and Frequency Control, vol. 50, pp. 419-427, 2003.

[29] P. Moilanen, "Ultrasonic guided waves in bone," IEEE Transactions on Ultrasonics, Ferroelectrics and Frequency Control, vol. 55, pp. 1277-1286, 2008.

[30] D. Trad, T. Ulrych and M. Sacchi, "Latest views of the sparse Radon transform," Geophysics, vol. 68, pp. 386-399, 2003.

[31] J. R. Thorson and J. F. Claerbout, "Velocity-stack and slant-stack stochastic inversion," Geophysics, vol. 50, pp. 2727-2741, 1985 .

[32] M. D. Sacchi and T. J. Ulrych, "High-resolution velocity gathers and offset space reconstruction," Geophysics, vol. 60, p. 1169, 1995.

[33] R. O. Schmidt, "Multiple emitter location and signal parameter estimation," IEEE Transactions on Antennas and Propagation, vol. 34, pp. 276-280, 1986.

[34] J. G. Minonzio, J. Foiret, M. Talmant, and P. Laugier, "Impact of attenuation on guided mode wavenumber measurement in axial transmission on bone mimicking plates," Journal of the Acoustical Society of America, vol. 130, pp. 3574-3582, 2011.

[35] M. Talmant, J. Foiret and J. G. Minonzio, "Guided waves in cortical bone," in Bone quantitative ultrasound Dordrecht, Heidelberg, London, New York: Springer, 2011, pp. 147-179.

[36] J. G. Minonzio, J. Foiret, P. Moilanen, J. Pirhonen, Z. Zhao, M. Talmant, J. Timonen, and P. Laugier, "A free plate model can predict guided modes propagating in tubular bone-mimicking phantoms," Journal of the Acoustical Society of America, vol. 137, pp. EL98-EL104, 2015.

[37] F. Lefebvre, Y. Deblock, P. Campistron, D. Ahite, and J. J. Fabre, "Development of a new ultrasonic technique for bone and biomaterials in vitro characterization," Journal of Biomedical Materials Research, vol. 63, pp. 441-446, 2002.

[38] P. Moilanen, Z. Zhao, P. Karppinen, T. Karppinen, V. Kilappa, J. Pirhonen, R. Myllylä, E. Hæggström, and J. Timonen, "Photo-acoustic Excitation and Optical Detection of Fundamental Flexural Guided Wave in Coated Bone Phantoms," Ultrasound in Medicine \& Biology, vol. 40, pp. 521-531, 2014.

[39] I. A. Viktorov, Rayleigh and Lamb waves: physical theory and applications: Plenum press New York, 1967.

[40] J. B. Harley and J. M. F. Moura, "Sparse recovery of the multimodal and dispersive characteristics of Lamb waves," Journal of the Acoustical Society of America, vol. 133, p. 2732, 2013.

[41] C. B. Park, "Imaging dispersion curves of surface waves on multi-channel record," SEG Technical Program Expanded Abstracts, vol. 17, p. 1377, 1999.

[42] K. Xu, J. G. Minonzio, D. Ta, B. Hu, W. Wang, and P. Laugier, "Sparse inversion SVD method for dispersion extraction Sparse SVD Method for High Resolution Extraction of the Dispersion Curves of Ultrasonic Guided Waves 30 
1 of ultrasonic guided waves in cortical bone," in 2015 6th European Symposium on Ultrasonic Characterization of Bone (ESUCB), 2 2015, pp. 1-3.

3 [43] M. D. Sacchi, "Reweighting strategies in seismic deconvolution," Geophysical Journal International, vol. 129, pp. 651$4656,1997$.

5 [44] T. N. Tran, L. H. Le and M. D. Sacchi, "High-Resolution Imaging of Dispersive Ultrasonic Guided Waves in Human Long 6 Bones Using Regularized Radon Transforms," in 5th International Conference on Biomedical Engineering in Vietnam, 2015, 7 pp. $28-31$.

8 [45] T. N. Tran, L. H. Le, M. D. Sacchi, V. Nguyen, and E. H. Lou, "Multichannel filtering and reconstruction of ultrasonic 9 guided wave fields using time intercept-slowness transform," Journal of the Acoustical Society of America, vol. 136, pp. 248$510 \quad 259,2014$.

[46] J. F. Claerbout, "Earth sounding analysis: Processing versus inversion," Blackwell Scientific Publications, Inc., 1992.

[47] P. C. Hansen and D. P. O'Leary, "The use of the L-curve in the regularization of discrete ill-posed problems," SIAM Journal on Scientific Computing, vol. 14, pp. 1487-1503, 1993.

[48] W. H. Press, Numerical recipes 3rd edition: The art of scientific computing: Cambridge university press, 2007. 


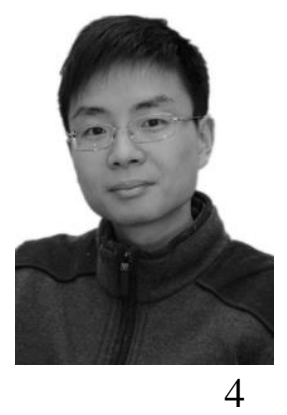

Kailiang Xu was born in Zhejiang, China, in 1985. He received the B.S. degree in electronic information science and technology and dual B.S. degree computer science from Inner Mongolia University in 2006. He then received the Ph.D. degree in biomedical engineering from Fudan University in 2012. His research interests are mainly focused on

5 ultrasound in biomedicine and NDE.

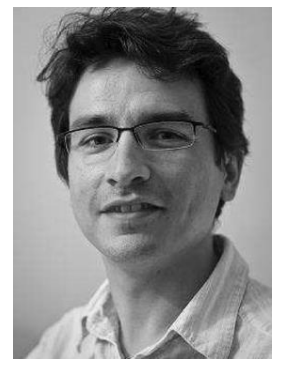

Jean-Gabriel Minonzio was born in Dijon, France, in 1978. He received the B.S. degree in engineering physics from Ecole Supérieure de Physique et de Chimie Industrielles de la ville de Paris (ESPCI), Paris, France, in 2003. He obtained the M.S. and Ph.D. degrees 10 in physical acoustics from University Denis Diderot, Paris, in 2003 and 2006, respectively.

signal processing, underwater acoustics, and target characterization. He currently works on elastic guided waves in cortical bone at the Laboratoire d'Imagerie Biomédicale (LIB), Universite Pierre et Marie Curie

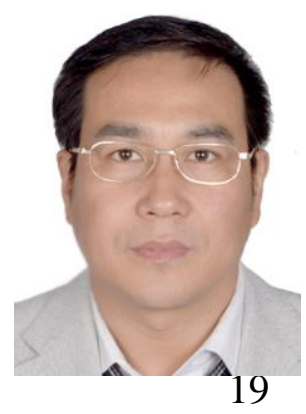

Dean Ta was born in China in 1972. He received $\mathrm{Ph}$. D degree from the Institute of Acoustics, Tongji University in May 2002. He was promoted to full professor in 2010. He is currently vice head of Electronic Eng Dept of Fudan University, vice-chairman of Biomed Ultrasound Eng Speciality of Chinese Soc of Acoustics and vice-chairman of 
1 journals including Ultrasound Med \& Biology. He was selected as superexcellent PostDoc at Fudan 2 University, the "Century Star" of Fudan Univ and super-excellent youth teacher program of university at

3 Shanghai in 2006. He was selected as Shanghai Rising-Star Program (2007), New Century Excellent 4 Talents of the Education Ministry of China (2010), The first "Wei Moan Acoustics Award" (2013) and 5 Outstanding young scientist grant of NSF China (2015). He was also the Principal Investigators of more 6 than 20 projects. In the last decade, he has contributed more than 160 papers, coauthored 3 books and 7 7 patents. His research interests include biomedical ultrasound and diagnosis system, and medical signal 8 processing. The generation, propagation and applications of ultrasonic guided waves in medicine (bone) 9 and NDT \& E. The applications of numerical techniques in ultrasound.

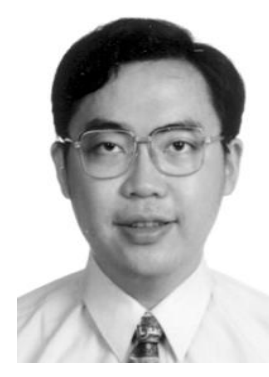

Bo $\mathrm{Hu}$ received the B.S. and Ph.D. degrees in electronic engineering from Fudan University, Shanghai, China, in 1990 and 1996, respectively. He is now a professor and Ph.D. advisor of electronic engineering at Fudan University, Shanghai. His research 14 interests include digital image processing, digital communication, and digital system 15 design.

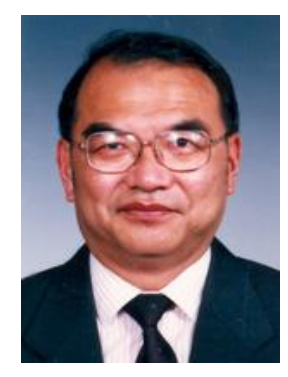

Weiqi Wang was born in Shanghai, China on May, 1939. He graduated from the Physics Department of Fudan University in 1961. He was elected to be a member of the Chinese Academy of Engineering in 1999. He is the executive professor and supervisor of the

20 Ph.D. program in Fudan University. He was the recipient of AIUM/WFUMB Pioneer Sparse SVD Method for High Resolution Extraction of the Dispersion Curves of Ultrasonic Guided Waves 33 
1 Award, National Invention Award (2nd place), and other 17 awards. His research interests include signal

2 processing, medical electronics, biomedical ultrasound and development of ultrasonic diagnosis system.

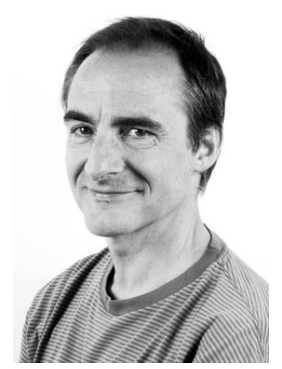

Pascal Laugier (M'10) was born in Paris, France. He is a graduate of the Ecole Normale Superieure de Cachan (France) and received a Ph.D. degree in physical acoustics from the University Paris 7, France, in 1987. He holds a full-time permanent position at the

$7 \quad$ National Center for Scientific Research (CNRS) as a Research Director. He is the head of

8 the Laboratoire d'Imagerie Biomedical (LIB), a public laboratory affiliated with the University Pierre et

9 Marie Curie-Paris 6, CNRS, and INSERM.

He co-authored 200 articles in peer-reviewed journals, and 14 patents, and co-edited a book entitled Bone Quantitative Ultrasound, published by Springer in 2011. His research activities are focused on the areas of biomedical applications of ultrasound. The team he leads has been involved for 20 years in sustained research in the field of bone quantitative ultrasound. His research centers on understanding the interaction between ultrasound and bone structures, using experiments, numerical simulations, and analytical models, and on developing innovative ultrasound-based technologies for in vivo bone assessment.

Dr. Laugier was awarded the CNRS Bronze Medal in 1993, the Yves Rocard Prize from the French Physics Society in 1998, the European Grand Prix for Innovation in 2001, and the Medal of the French Acoustical Society in 2009. He was elected a Member of the European Academy of Sciences in 2003. He 19 is a Fellow of the American Institute for Medical and Biological Engineering (AIMBE), a Fellow of the Acoustical Society of America (ASA), and an Honorary Fellow of the American Institute of Ultrasound in Sparse SVD Method for High Resolution Extraction of the Dispersion Curves of Ultrasonic Guided Waves 34 


\section{Page 35 of 39}

1

2

31 Medicine. He is an associate editor of the IEEE Transactions on Ultrasonics Ferroelectrics and Frequency

4

7

$\varepsilon$

9

10

11

12

13

14

15

16

17

18

19

20

21

22

23

24

25

26

27

28

29

30

31

32

33

34

35

36

37

38

39

40

41

42

43

44

45

46

47

48

49

50

51

52

53

54

55

56

57

58

59

60

2 Control and a member of the editorial boards of Ultrasound in Medicine and Biology, Ultrasonic Imaging,

3 and two other professional journals.

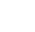

(4)

(1)

(1)

.

(3)

.

(5)

(2)




\section{2 - Variable definition}

3 Input:

- $\quad M(R, E, t):$ 3-D measurement matrix obtained by a multi-emitter (E) and multi-receiver (R) system.

$5 \quad-\quad N_{k}, N_{f}:$ number of points on the wavenumber and frequency axes.

$6-N_{r}$ : number of highest singular values associate to the signal space, it will be used to obtain the $U_{R}$ consisting of

$7 \quad N_{r}$ singular vectors. A flexible rank strategy can be employed by using a threshold to select the highest singular

$8 \quad$ values and corresponding singular vectors.

$9 \quad-\quad n_{\max }$ : maximum iteration times, an heuristic value is 20.

$10-\sigma$ : hyperparameter, an heuristic value is in the range of $0.01 \sim 0.1$.

- $\quad \mu$ : Lagrange factor, also named as regularization parameter or damping parameter.

- $\quad J$ : cost function.

$13-\xi$ : threshold of the relatively convergence difference of the cost function. An heuristic value is 0.02 .

Output:

- $\widetilde{U}:$ a $N_{k} \times N_{k}$ matrix remains the adjusted singular vectors.

- $\quad \operatorname{Norm}(k, f)$ : estimated Norm function.

2-D FT projecting the $M(R, E, t)$ into the $W(k, E, f)$ space; 
$1 \quad$ for each $f_{p} \in f\left(1,2, \ldots, N_{f}\right)$

$2 \quad$ a) SVD decomposition, $[U, S, V]=S V D\left[W\left(k, E, f_{p}\right)\right]$

3 b) Obtaining the modified $U_{R}$ by discarding the insignificant singular vectors and normalizing the $N_{r}$ highest

$4 \quad$ singular values to enhance the weak modes;

5 c) LS-SVD Initialization:

6 According to Eq. (8), the LS-SVD estimation is following

$\mu_{t}=\mu \operatorname{trace}\left[\left(U_{R}^{-1}\right)^{H} U_{R}^{-1}\right]$

$\widetilde{U}^{(0)}=\left[\left(U_{R}{ }^{-1}\right)^{H} U_{R}{ }^{-1}+\mu_{t} I\right]^{-1}\left(U_{R}{ }^{-1}\right)^{H} ;$

$\widetilde{W}^{(0)}\left(k, E, f_{p}\right)=\widetilde{U}^{(0)} S V^{H} ;$

d) S-SVD procedure:

$>$ Initialization with the $N_{k} \times N_{k}$ Toeplitz matrix $Q$ :

If using the $l_{1}$-norm, then

$$
Q^{\prime}=\left(\begin{array}{ccc}
{\left[\sum_{j=1}^{N_{E}}\left(\left|\widetilde{W}^{(0)}\left(1, j, f_{p}\right)\right|+\sigma^{2}\right)\right]^{-1}} & \ldots & 0 \\
\ldots & \ddots & \ldots \\
0 & \ldots & {\left[\sum_{j=1}^{N_{E}}\left(\left|\widetilde{W}^{(0)}\left(N_{k}, j, f_{p}\right)\right|+\sigma^{2}\right)\right]^{-1}}
\end{array}\right)
$$

If using the Cauchy norm, then

$$
\begin{aligned}
& Q^{\prime \prime}=\left(\begin{array}{ccc}
{\left[\sum_{j=1}^{N_{E}}\left(\left|\widetilde{W}^{(0)}\left(1, j, f_{p}\right)\right|^{2}+\sigma^{2}\right)\right]^{-1}} & \ldots & 0 \\
\cdots & \ddots & \ldots \\
0 & \ldots & {\left[\sum_{j=1}^{N_{E}}\left(\left|\widetilde{W}^{(0)}\left(N_{k}, j, f_{p}\right)\right|^{2}+\sigma^{2}\right)\right]^{-1}}
\end{array}\right) ; \\
& J^{(0)}=\mu\left\|W\left(k, E, f_{p}\right)\right\|_{F}^{2} ;
\end{aligned}
$$


1 Let $\Delta J=\infty$ to ensure of the first iteration;

$2>$ Reweighting iteration:

$3 \quad$ While $\left(n<n_{\max }\right) \&(\Delta J>\xi)$

4 1. Updating the estimated $\widetilde{U}$ and $\widetilde{W}$

$5 \quad \mu_{t}=\mu \operatorname{trace}\left[\left(U^{(n)^{-1}}\right)^{H} U^{(n)^{-1}}\right]$

$6 \quad \widetilde{U}^{(n+1)}=\left[\left(U^{(n)^{-1}}\right)^{H} U^{(n)^{-1}}+\mu_{t} Q\right]^{-1}\left(U^{(n)^{-1}}\right)^{H} ;$

$7 \quad \widetilde{W}^{(n+1)}\left(k, E, f_{p}\right)=\widetilde{U}^{(n+1)} S V^{H} ;$

2. Updating the reweighting matrix $N_{k} \times N_{k} Q$

If using the $l_{1}$-norm, then

$Q^{\prime}=\left(\begin{array}{ccc}{\left[\sum_{j=1}^{N_{E}}\left(\left|\widetilde{W}^{(n+1)}\left(1, j, f_{p}\right)\right|+\sigma^{2}\right)\right]^{-1}} & & 0 \\ \ldots & \cdots & \ldots \\ 0 & \ddots & \ldots \\ & \ldots & {\left[\sum_{j=1}^{N_{E}}\left(\left|\widetilde{W}^{(n+1)}\left(N_{k}, j, f_{p}\right)\right|+\sigma^{2}\right)\right]^{-1}}\end{array}\right)$

If using the Cauchy norm, then

$$
Q^{\prime \prime}=\left(\begin{array}{ccc}
{\left[\sum_{j=1}^{N_{E}}\left(\left|\widetilde{W}^{(n+1)}\left(1, j, f_{p}\right)\right|^{2}+\sigma^{2}\right)\right]^{-1}} & & 0 \\
\cdots & \cdots & \cdots \\
0 & \ddots & \cdots \\
& \cdots & {\left[\sum_{j=1}^{N_{E}}\left(\left|\widetilde{W}^{(n+1)}\left(N_{k}, j, f_{p}\right)\right|^{2}+\sigma^{2}\right)\right]^{-1}}
\end{array}\right)
$$

3. Computing the cost function

If using the $l_{1}$-norm, then

$$
J^{(n+1)}=\left\|\widetilde{U}^{(n+1)^{-1}} \widetilde{W}^{(n+1)}\left(k, E, f_{p}\right)-S V^{H}\right\|_{F}^{2}+\mu \sum_{i=1}^{N_{k}}\left(\sigma^{2}+\sum_{j=1}^{N_{E}}\left|\widetilde{W}^{(n+1)}\left(i, j, f_{p}\right)\right|^{2}\right)
$$

If using the Cauchy norm, then 
1

2

$\begin{array}{ll}3 & 1\end{array}$

4

5

62

7

8

9

103

11

12

134

14

15

16

17

18

19

20

21

22

2

24

25

$26 \quad 8$

27

28

29

30

31

32

33

34

35

36

37

38

39

40

41

42

43

44

45

46

47

48

49

50

51

52

53

54

55

56

57

58

59

60

$$
J^{(n+1)}=\left\|\widetilde{U}^{(n+1)^{-1}} \widetilde{W}^{(n+1)}\left(k, E, f_{p}\right)-S V^{H}\right\|_{F}^{2}+\mu \sum_{i=1}^{N_{k}} \ln \left(1+\frac{\sum_{j=1}^{N_{E}}\left|\widetilde{W}^{(n+1)}\left(i, j, f_{p}\right)\right|^{2}}{\sigma^{2}}\right)
$$

4. Calculating the relative iteration convergence difference

$$
\Delta J=2\left|J^{(n+1)}-J^{(n)}\right| /\left(J^{(n+1)}+J^{(n)}\right) ;
$$

5. $n=n+1$

end;

e) Summing the estimated norm function;

$7 \quad$ end $f_{p}$

Return $\operatorname{Norm}(k, f)$. 